\title{
CORRIGENDUM \\ Combining experimental evolution with next-generation sequencing: a powerful tool to study adaptation from standing genetic variation
}

\author{
C Schlötterer, R Kofler, E Versace, R Tobler and SU Franssen
}

Heredity (2016) 116, 248; doi:10.1038/hdy.2015.85

Correction to: Heredity (2015) 114, 431-440; doi:10.1038/hdy.2014.86

The authors would like to note a correction to Box 1: 'In the following, two types of genetic loci are referred to as strong effect loci

\footnotetext{
Box 1 Recommendations for experimental design

In the following, two types of genetic loci are referred to as strong effect loci (SEL) and weak effect loci (WEL) that have selection coefficients of 0.1 and $\leqslant 0.025$ respectively.

The experimental population

Duration of experiment: minimum of 10-20 generations for SEL and $>100$ for WEL.

Experimental population size: minimum of 500 individuals for SEL and $>1000$ for WEL.

Number of replicates: minimum of 3 for SEL and $>10$ for WEL.

Number of distinct chromosomes in the starting population: the larger the better. General remark: given budget constraints, it is difficult to increase both the population size and the number of replicates. In this case, we favor increased replication over larger population sizes, because this increases the power to identify strongly selected loci while leaving weakly selected loci unaffected. Moreover, replication acts as a buffer against accidental population loss, for example because of a bottleneck or viral infection.
}

Sequencing of pooled individuals (Pool-Seq)

Pool size: maximize the number of individuals in the Pool.

Coverage: minimum of $50 \times$ for SEL and $>200 \times$ for WEL.

Read type: only paired-end with at least $100 \mathrm{bp}$ read length.

\section{Recommendations for data analysis}

Trimming: reads should be trimmed to remove poor-quality bases (base quality $<20$ ).

Reference genome: use the conspecific reference genome or that of a closely related species; note however that even closely related species may cause considerable biases.

Mapping: allow for gaps, avoid seeding, align the entire read (semiglobal alignment) and take advantage of the second read by realigning unmapped mates.

Filtering: remove broken pairs, only use reads with mapping quality $>20$, remove positions flanking indels, remove duplicate reads, mask repetitive sequences and potential copy number variants and treat low recombining regions separately.

Test statistic: properties of novel test statistics should be tested by computer simulations using software tools like MimicrEE (Kofler and Schlötterer, 2014) or forqs (Kessner and Novembre, 2014a).
(SEL) and weak effect loci (WEL) that have selection coefficients of 0.1 and $\leqslant 0.25$ respectively' should read 'In the following, two types of genetic loci are referred to as strong effect loci (SEL) and weak effect loci (WEL) that have selection coefficients of 0.1 and $\leqslant 0.025$ respectively'. A correct version of Box 1 appears below: 